\title{
Integrative Proteomic Characterization of Trace \\ FFPE Samples in Early-Stage Gastrointestinal \\ Cancer
}

\section{Lingling Li}

Fudan University https://orcid.org/0000-0002-7760-3446

Hui Liu

Henan Normal University

Yan Li

Fudan University

Chunmei Guo

Fudan University

Bing Wang

Henan Normal University

Dan Shen

Fudan University

Qiao Zhang

Fudan University

Chen Ding ( $\nabla$ chend@fudan.edu.cn )

Fudan University

\section{Research}

Keywords: Early-stage gastrointestinal cancer, proteomics, 10,000 phosphosites, immune infiltration, kinases characterization

Posted Date: December 3rd, 2021

DOI: https://doi.org/10.21203/rs.3.rs-1084524/v1

License: (c) (i) This work is licensed under a Creative Commons Attribution 4.0 International License. Read Full License

Version of Record: A version of this preprint was published at Proteome Science on April 9th, 2022. See the published version at https://doi.org/10.1186/s12953-022-00188-0. 


\section{Integrative proteomic characterization of trace FFPE samples in}

\section{early-stage gastrointestinal cancer}

Lingling $\mathrm{Li}^{1}$, Hui Liu ${ }^{2}$, Yan $\mathrm{Li}^{1}$, Chunmei Guo ${ }^{1}$, Bing Wang ${ }^{2}$, Dan Shen ${ }^{1}$, Qiao Zhang ${ }^{1}$ and Chen $\operatorname{Ding}^{1,2,3, *}$

* Correspondence: chend@fudan.edu.cn (C.D.).

${ }^{1}$ State Key Laboratory of Genetic Engineering and Collaborative Innovation Center for Genetics and Development, School of Life Sciences, Institute of Biomedical Sciences, Human Phenome Institute, Fudan University, Shanghai 200433, China;

${ }^{2}$ State Key Laboratory Cell Differentiation and Regulation, Overseas Expertise Introduction Center for Discipline Innovation of Pulmonary Fibrosis, (111 Project), College of Life Science, Henan Normal University, Xinxiang, Henan 453007, China.

${ }^{3}$ Academy of Medical Science, Zhengzhou University, Zhengzhou 450052, China.

Full list of author information is available at the end of the article. 


\begin{abstract}
Background: The surveillance and therapy of early-stage cancer would be better for patients' prognosis. However, the extreme trace amount of tissue samples in different stages have limited in portraying the characterization of early-stage cancer. Therefore, we focused on and presented comprehensive proteomic and phosphoproproteomic profiling of the trace FFPE samples from early-stage gastrointestinal cancer, and then explored the potential biomarkers of early-stage gastrointestinal cancer.
\end{abstract}

Methods: In this study, a quantitative proteomic method with chromatography with mass spectrometry (LC-MS/MS) was used to analyse the proteomic difference between the trace early-stage esophageal squamous cell carcinoma (EESCC) and early-stage duodenum adenocarcinoma cancer (EDAC).

Results: We identified $\sim 6,000$ proteins and $>10,000$ phosphosites in single trace FFPE samples. The distinct separation of EESCC and EDAC illustrated the functions of cell cycle (RB1 T373, EGFR T693) in EESCC, and the positive impacts of apoptosis, metabolic processes (MTOR and MTOR S1261) in EDAC. Furthermore, we deconvoluted the immune infiltration of early-stage gastrointestinal cancer, in which higher immune cell signatures were detected in EDAC, and showed the specific cytokines in EESCC and EDAC. We performed kinases-substates relationship analysis and elucidated the specific proteomic kinase characterization of EESCC and EDAC, and proposed the medicative effects and corresponding drugs for EESCC and EDAC at the clinic.

Conclusion: We disclosed the specific immune characterization of the early-stage gastrointestinal cancer, and presented potential makers of EESCC (EGFR, PDGFRB, CDK4, WEE1) and EDAC (MTOR, MAP2K1, MAPK3). This study represents a major stepping stone towards investigating the carcinogenesis mechanism of gastrointestinal cancer, and providing a rich resource for medicative strategy in the clinic.

\title{
Key Words:
}

Early-stage gastrointestinal cancer, proteomics, 10,000 phosphosites, immune infiltration, kinases characterization 


\section{Introduction}

Cancer is still a major health problem worldwide, leading nearly 10 million deaths every year $[1,2]$. Surgery is the predominant curative treatment strategy in advanced stages (T2 to T4 stages), with poor quality of life (QOL) and low five-year survival rate $(<30 \%)$ [3]. Early screening and diagnosis of disease, approved by World Health Organization (WHO) has been prominent nowadays, especially in gastrointestinal cancer. In addition, advances of endoscopic submucosal dissection (ESD) [4] have achieved the early detection cancers (T1 stage), especially in gastrointestinal cancer, with higher QOL and significantly improved overall survival rate (> 90\%) [5-7]. The major events in advanced-stage cancer, appeared to be identified in as early as in early-stage cancer, thus, the surveillance and therapy of early-stage cancer would be better for patients' prognosis. However, the extreme trace amount of tissue samples in different stages have limited in portraying the characterization of early-stage cancer.

Pathologically, formalin-fixed, paraffin-embedded (FFPE) biospecimens represent gold standard for archiving pathology samples, keeping the tissues stability, providing a valuable resource for clinical and biomarker researches [8]. In addition, FFPE tissue biopsies showed a high degree of the proteome pattern similarity between histological regions samples collected for 1 and 15 years, and facilitates tumor stratification [9]. During the last decade, great progress has been made in mass spectrometry-based proteomics, providing chances for employing biobanked FFPE samples to many types of cancers to reveal cancer proteomic characterization. Specifically, proteomic analysis of colon rectal cancer (CRC) revealed decreased T cell infiltration and increased glycolysis in CRC [10]; Sai Ge et.al, described a proteomic landscape of diffuse-type gastric cancer, and illustrated the overrepresentation of immune response in the third subtypes with worst survival [11]. However, the proteomic profiling of trace FFPE samples in early-stage cancer remain largely unknow.

Here, we collected FFPE samples of early-stage gastrointestinal cancer, including early-stage esophageal squamous cell carcinoma (EESCC) and early-stage duodenum adenocarcinoma cancer (EDAC). We presented a comprehensive proteomic landscape of early-stage gastrointestinal cancer, with the identification of $\sim 6,000$ proteins and $>10,000$ phosphosites in single trace FFPE samples. We revealed the functional classification and proteomic characterization of EESCC and EDAC. In addition, we elucidated the immune infiltration, cytokines types, specific kinases of EESCC and EDAC, and proposed the potential kinase clinic strategy for EESCC and EDAC, providing a novel useful resource for potential therapeutic approaches for gastrointestinal cancer.

\section{Materials and methods}

\section{Processing of formalin-fixed, paraffin-embedded (FFPE) specimens}

In this study, all the tissue samples from the corresponding substages were separately dissected from the formalin-fixed, paraffin-embedded (FFPE) slides, and were prepared and provided by Zhongshan Hospital, Fudan University. The study was carried out in compliance with the ethical standards of Helsinki Declaration II and approved by the Institution Review Board of Fudan University Zhongshan Hospital (B2019-200R). For clinical sample preparation, slides (10 $\mu \mathrm{m}$ thick) from FFPE blocks were macro-dissected, deparaffinized with xylene and washed with ethanol. All the selected specimens were evaluated and confirmed by two or three experienced and board-certified gastrointestinal pathologists, and materials were aliquoted and kept in storage at $-80^{\circ} \mathrm{C}$ until further processing.

\section{Protein extraction and digestion}

Nearly $50 \mu \mathrm{L}$ TCEP buffer (2\% deoxycholic acid sodium salt, $40 \mathrm{mM}$ 2-chloroacetamide, $100 \mathrm{mM}$ tris- 
phosphine hydrochloride, $10 \mathrm{mM}$ (2-carboxyl)-phosphine hydrochloride, $1 \mathrm{mM}$ phenylmethylsulfonyl fluoride mixed with MS water, $\mathrm{PH} 8.5)$ was added into $1.5 \mathrm{~mL}$ EP tubes with prepared samples $(0.01 \mathrm{~cm}$ $\times 0.01 \mathrm{~cm}$ ), and then heated in a $99^{\circ} \mathrm{C}$ metal bath for $30 \mathrm{~min}$. Cool to room temperature, $3 \mu \mathrm{g}$ trypsin (REF: V528A, PROMEGA) was added into each tube and digested for 18 hours in a $37^{\circ} \mathrm{C}$ incubator. Then, $13 \mu \mathrm{L} 10 \%$ formic acid was added into each tube and made vortex for $3 \mathrm{~min}$, and then sedimentation for $5 \min (12,000 \mathrm{~g})$. After that, a new $1.5 \mathrm{~mL}$ tube with $350 \mu \mathrm{L}$ buffer $(0.1 \%$ formic acid in 50\% acetonitrile) is needed for collected the supernatant for extraction (vortex for $3 \mathrm{~min}$, and then $12,000 \mathrm{~g}$ sedimentation for $5 \mathrm{~min}$ ). And then the supernatant was transferred into a new tube for drying in $60{ }^{\circ} \mathrm{C}$ vacuum drier. After drying, $100 \mu \mathrm{L} 0.1 \%$ formic acid was needed for dissolving the peptides and vortex for $3 \mathrm{~min}$, and then sedimentation for $3 \mathrm{~min}(12,000 \mathrm{~g})$. The supernatant was picked into new tube and then desalinated. Before desalination, the activation of pillars with 2 slides of $3 \mathrm{M} \mathrm{C} 8$ disk is required, and the lipid is as follows: $90 \mu \mathrm{L} \mathrm{100 \%}$ acetonitrile twice, $90 \mu \mathrm{L} 50 \%$ and $80 \%$ acetonitriler once in turn, and then $90 \mu \mathrm{L} \mathrm{50 \%}$ acetonitrile once. After pillar balance with $90 \mu \mathrm{L} 0.1 \%$ formic acid twice, the supernatant of the tubes was loading into the pillar twice, and decontamination with $90 \mu \mathrm{L} 0.1 \%$ formic acid twice. Lastly, $90 \mu \mathrm{L}$ elution buffer ( $0.1 \%$ formic acid in $50 \%$ acetonitrile) was added into the pillar fir elution twice and only the effluent was collected for MS. And then the collect of lipid was put in $60{ }^{\circ} \mathrm{C}$ vacuum drier for drying.

\section{Proteome analysis in LC-MS/MS Analysis}

For the proteomic profiling of samples, peptides were analyzed on a Q Exactive HF-X Hybrid Quadrupole-Orbitrap Mass Spectrometer (Thermo Fisher Scientific, Rockford, IL, USA) coupled with a high-performance liquid chromatography system (EASY nLC 1200, Thermo Fisher). Dried peptide samples re-dissolved in Solvent A (0.1\% FA in water) were loaded to a 2-cm self-packed trap column (100- $\mu \mathrm{m}$ inner diameter, $3 \mu \mathrm{m}$ ReproSil-Pur C18-AQ beads, Dr. Maisch $\mathrm{GmbH}$ ) using Solvent A and separated on a $150-\mu \mathrm{m}$-inner-diameter column with a length of $30 \mathrm{~cm}(1.9 \mu \mathrm{m}$ ReproSil-Pur C18-AQ beads, Dr. Maisch $\mathrm{GmbH}$ ) over a $150 \mathrm{~min}$ (EESCC and EDAC). The eluted peptides were ionized under $2.0 \mathrm{kV}$ and introduced into mass spectrometer). MS was performed under a data-dependent acquisition mode. For the MS1 Spectra full scan, ions with $\mathrm{m} / \mathrm{z}$ ranging from 300 to 1,400 were acquired by Orbitrap mass analyzer at a high resolution of 120,000 . The automatic gain control (AGC) target value was set as 3E6. The maximal ion injection time was $80 \mathrm{~ms}$. MS2 Spectra acquisition was performed in the ion trap mode at a rapid speed. Precursor ions were selected and fragmented with higher energy collision dissociation (HCD) with a normalized collision energy of $27 \%$. Fragment ions were analyzed by the ion trap mass analyzer with the AGC target at 5E4. The maximal ion injection time of MS2 was 20 ms. Peptides that triggered MS/MS scans were dynamically excluded from further MS/MS scans for $12 \mathrm{~s}$.

\section{Proteomics Data Processing and Analyzing}

All profiling data qualified were downloaded from firmiana platform (https://phenomics.fudan.edu.cn/firmiana/) against the human RefSeq protein database (updated on 0407-2013) in the National Center for Biotechnology Information (NCBI). Student's $t$ test was used for statistical analysis, and data from more than two groups were analyzed by one-way ANOVA in SPSS Statistics 19.0 and a subsequent Fisher's least significant difference $t$ test. Results were considered significantly when $p<0.05$.

The maximum number of missed cleavages was set to 2. A mass tolerance of $20 \mathrm{ppm}$ for precursor and $0.5 \mathrm{Da}$ for production was allowed. The fixed modification was cysteine carbamidomethylation and the variable modifications were $\mathrm{N}$-acetylation and oxidation of methionine. For the quality control of 
proteins identification, the target-decoy based strategy was applied to confirm the FDR (False Discovery Rate) of both peptide and protein was lower than $1 \%$. Percolator was used to obtain the probability value (q-value), validating the FDR (measured by the decoy hits) of every peptide-spectrum match (PSM) was lower than $1 \%$. Then all the peptides shorter than seven amino acids were removed. The cutoff ion score for peptide identification was 20. All the PSMs in all fractions were combined for protein quality control, which was a stringent quality control strategy. The q-values of both target and decoy peptide sequences were dynamically increased until the corresponding protein FDR was less than $1 \%$ employing the parsimony principle. Finally, to reduce the false positive rate, the proteins with at least one unique peptide were selected for further investigation.

\section{Phosphopeptide enrichment and analysis}

The phosphoproteome samples were prepared by Fe-NTA Phosphopeptide Enrichment Kit (Thermo, Catalog: A32992) according to the manufacturer's instruction. The peptides were resuspended in $200 \mu \mathrm{L}$ binding/wash buffer and loaded to the equilibrated spin column. The resin was mixed with the sample by gently tapping. The mixture was incubated for $30 \mathrm{~min}$ and centrifuged at $1,000 \times \mathrm{g}$ for $30 \mathrm{~s}$ to discard the flowthrough. The column was then washed by $200 \mu \mathrm{L}$ of binding/wash buffer and centrifuged at $1,000 \times \mathrm{g}$ for $30 \mathrm{~s}$ for 3 times and washed by $200 \mu \mathrm{L}$ of LC-MS grade water for one additional time. The phosphopeptide was eluted by adding $100 \mu \mathrm{L}$ of elution buffer and centrifuged at $1,000 \times \mathrm{g}$ for $30 \mathrm{~s}$ for 2 times. Phosphopeptides were dried down for LC-MS/MS analysis.

For the phosphoproteomic analysis, the phosphopeptides were analyzed on Orbitrap Fusion Lumos Tribrid Mass Spectrometer (Thermo Fisher Scientific, Rockford, IL, USA) equipped with an Easy nLC1000 (Thermo Fisher Scientific, Rockford, IL, USA) and a Nanoflex source (Thermo Fisher Scientific, Rockford, IL, USA). Dried peptide samples re-dissolved in Solvent A (0.1\% FA in water) were loaded to a 2-cm self-packed trap column using Solvent A and separated on a 150- $\mu \mathrm{m}$-inner-diameter column with a length of $30 \mathrm{~cm}$ over a 150 min gradient (buffer A: $0.1 \%$ FA in water; buffer B: $0.1 \%$ FA in $80 \%$ $\mathrm{ACN})$ at a constant flow rate of $600 \mathrm{~nL} / \mathrm{min}(0-150 \mathrm{~min}, 0 \mathrm{~min}, 4 \% \mathrm{~B} ; 0-10 \mathrm{~min}, 4-15 \% \mathrm{~B} ; 10-125 \mathrm{~min}$, $15-30 \% \mathrm{~B} ; 125-140 \mathrm{~min}, 30-50 \% \mathrm{~B} ; 140-141 \mathrm{~min}, 50-100 \% \mathrm{~B} ; 141-150 \mathrm{~min}, 100 \% \mathrm{~B}$ ). The eluted phosphopeptides were ionized and detected. Mass spectra were acquired over the scan range of $\mathrm{m} / \mathrm{z} 350$ 1500 at a resolution of 120,000 (AUG target value of 5E5 and max injection time $50 \mathrm{~ms}$ ). For the MS2 scan, the higher-energy collision dissociation fragmentation was performed at a normalized collision energy of $30 \%$. The MS2 AGC target was set to $1 \mathrm{E} 4$ with a maximum injection time of $10 \mathrm{~ms}$, Peptide mode was selected for monoisotopic precursor scan, and charge state screening was enabled to reject unassigned $1+, 7+, 8+$, and $>8+$ ions with a dynamic exclusion time of $45 \mathrm{~s}$ to discriminate against previously analyzed ions between $\pm 10 \mathrm{ppm}$.

\section{Principal component analysis (PCA) of trace FFPE samples}

We performed PCA on a total of proteins/phosphoproteins identified in 6 gastrointestinal cancer sample to illustrated the global proteomic difference between EESCC and EDAC. The PCA function under the scikit-learn $\mathrm{R}$ package was implemented for unsupervised clustering analysis with the parameter ' $\mathrm{n} \_$components $=2$ ' on the expression matrix of global proteomic data. A colored ellipse represented the $95 \%$ confidence coverage for each group.

\section{Biological pathways enrichment analysis}

To investigate the dominant signaling pathways of the overlap proteins and each concentrated gradient of trace samples, we used gens sets of molecular pathways in DAVID [12]. For this analysis, pathways from the GOBP/KEGG database were considered. Statistical significance was considered when $P$ value 
was less than 0.05 and FDR $q$ value was no more than 0.1 .

\section{Kinase Activity Prediction and Phosphopeptide Analysis}

The phospho-proteome data of 6 samples were searched against the same database with MaxQuant. The phosphorylation of S or $\mathrm{T}$ or $\mathrm{Y}$ was set as variable modification, in which three mis-cleavages were allowed, with a minimum Andromeda score of 40 for spectra matches. The ratios of identified phosphorylation sites of all samples were used to estimate the kinase activities by Kinase-Substrate Enrichment Analysis (KSEA) algorithm [13]. The information of kinase-substrate relationships was obtained from publicly available databases, including PhosphoSite [14], Phospho.ELM [15] and PhosphoPOINT [16]. The information of substrate motifs was obtained either from the literature [17] or from an analysis of the KSEA dataset with Motif (sP) [18]. The kinase-substrate-motif network analysis was referenced from PhosphoSitePlus (PSP, https://www.phosphosite.org/homeAction) [19]. Statistical analysis was performed in $\mathrm{R}$ (version 3.5.1) with Kruskal-Wallis test.

\section{Results}

\section{Overview of proteomic landscape of trace FFPE samples in EESCC and EDAC}

To characterize the comprehensive proteomic landscape of trace FFPE samples, we collected proteomics and phosphoproteomics data from 3 early-stage ESCC (EESCC) patients and 3 early-stage DC (EDAC) patients who had not experienced prior chemotherapy or radiotherapy. A schematic of the experimental design is shown in Fig. 1a and Supplementary Fig. S1a. Proteomic analysis was performed on the basis of mass spectrometry (MS)-based label-free quantification strategy [11, 20]. Protein abundance of all samples was firstly calculated by intensity-based absolute quantification (iBAQ) [21, 22] and then normalized as a fraction of the total (FOT), allowing for comparisons between experiments.

At the protein level, identification of $\sim 6,000$ proteins were observed in single trace FFPE sample, and a total of 6,450 and 6,789 proteins (3 cases) were identified in the EESCC and EDAC, respectively (Supplementary Fig. S1b - S1d). The reference proteome was highly dynamic based on the protein abundance (FOT), which spanned over eight orders of magnitude. As well as in EDAC, the top proteins extensively expressed in EESCC were HBB, HBA1, HBD, ACTB, ALB, GSTP1, etc. (Fig. 1b - 1d). On the contrary, the low abundance proteins were different between EESCC and EDAC. Specifically, EPHA3, EPHB1, BTK, GNB3, TRIO, WDFY4 were detected in EESCC (Fig. 1b; Supplementary Table S1). In EDAC, low abundance proteins included TTN, DCHS2, MYCBP2, SGK223, WAPHAL, ARID2, etc. (Fig. 1c). Additionally, ESCC biomarkers identified in previous study, such as ACTA2, ANXA1 [23], HSPA9, THBS1 [24] etc. were also detected in EESCC, indicating the key events in advanced-stage cancer happened as earlier as in the early-stage cancer.

At the phosphoprotein level, identification of over 10,000 phosphosites was observed in the single trace FFPE sample. A total of 18,072 phosphosites corresponding to 4,173 phosphoproteins, and 12,200 phosphosites corresponding to 3,390 phosphoproteins were identified in EESCC and EDAC, respectively (Fig. 1e - 1g). The top abundance phosphoproteins covering HSPB1 (S82), KRT5 (S75), KRT13 (S427), KRT14 (S435), etc., and the low abundance phosphoproteins containing CUL4A (S10), INTS3 (S537), ABHD16A(S41), WDR59 (S564), SEC14L1 (T234), etc., were identified in EESCC (Fig. 1e; Supplementary Table S2). In EDAC, high phosphorylation of STK S4, THRAP3 S682, TMPO T74, CFL1 S75 and FLNA S3, and low phosphorylation of LIMA1 S692, PKN2 S535, BRPF1 S238, MAP2 S285, and TNS1 S899/S907 were disclosed (Fig. 1f). Overall, we established a comprehensive landscape of trace FFPE samples in early-stage gastrointestinal cancer (EESCC and EDAC) at multi-omics levels, and identified $\sim 6,000$ proteins and $>10,000$ phosphosites in single FFPE samples. 


\section{Proteomic characterization of EESCC and EDAC}

To investigate the correlations between the proteome and phosphoproteome of EESCC and EDAC, we performed integration analysis and found significant association between proteome $(\mathrm{n}=6,450)$ and phosphoproteome $(n=4,173)$ in EESCC (Pearson correlation, $R=0.16, p=9.5 E-15)$ (Supplementary Fig. S2a). As well, notably association between proteome $(n=6,789)$ and phosphoproteome $(n=3,390)$ in EDAC (Pearson correlation, $R=0.12, p=1.7 E-7)$ (Supplementary Fig. S2b), which allowed us to further investigating the characterizations of early-stage cancer.

Spearman's correlation showed lower coefficients (mean $=0.62$ ) between EESCC and EDAC than the same cancer type (mean $=0.74(\mathrm{ESCC})$ and $0.78(\mathrm{EDAC})$ ) indicated the difference in gastrointestinal cancer (Fig. 2a). To explore the difference between EESCC and EDAC, we performed principal component analysis (PCA) at the protein and phosphoprotein levels. Visualization of PCA differentiated the proteome profiles between EESCC and EDAC, as well as at the phosphoprotein level (Fig. 2b). The distinct separation suggested the fundamental difference between EESCC and EDAC.

The significance analysis of microarray (SAM) [25] was performed to investigate the characteristics of EESCC and EDAC at the protein level, which identified 791 differentially expressed proteins (DEPs) between EESCC and EDAC (t-test, $p<0.05$, fold change (FC) (EDAC/EESCC) $\geq 2$ or $\leq 0.5$ ), including 678 elevated (EDAC-proteins) and 113 descend proteins (EESCC-proteins) (Fig. 2c). GO-enrichment analysis presented that the EESCC-proteins were related to the primary functions of esophagus tissues, including keratinization ( $p=2.0 \mathrm{E}-4$ ) (e.g., CDH1, KRT2, etc.), cell division ( $p=1.2 \mathrm{E}-3)$ (e.g., NCAPD2, MCMBP, etc.), and epidermis development ( $p=1.7 \mathrm{E}-3$ ) (e.g., TCHH, CETN2, etc.) (Fig. 2d). The EDAC-proteins participated in Mapk signaling ( $p=7.7 \mathrm{E}-3)$ (e.g., MAPK1, MAPK3, etc.) and metabolic processes $(p=2.0 \mathrm{E}-4)$ (e.g., ACO2, PAK1, etc.), covering tricarboxylic acid cycle (TCA) ( $p=2.6 \mathrm{E}-9)$ (e.g., SDHA, IDH1, etc.), fatty acid-beta-oxidation ( $p=3.6 \mathrm{E}-8)$ (e.g., ACOX2, CPT2, etc.), ATP biosynthesis process ( $p=6.5 \mathrm{E}-6)$ (e.g., ATP5B, ATP5S, etc.), canonical glycolysis $(p=2.8 \mathrm{E}-4)$ (e.g., PFKL, TPI1, etc.) (Fig. 2d).

Observation of the difference between EESCC and EDAC was also identified at the phosphoprotein level (Supplementary Fig. S2c). For example, the phosphorylation of keratinization-/epidermis development-/cell division- related phosphoproteins were detected in EESCC, such as KRT2 S26, KRT5 S571, CDH3 T694, TGM3 S471, NCAPD2 S13, MCMBP S298, etc. In addition, the phosphorylation of MAPK T185/Y187, MAPK3 T202, ACO2 S79, ATP5B T140, PFKL S763, etc. was elevated in EDAC (Fig. 2e - 2f). Furthermore, we found the EESCC-phosphoproteins were associated in cell cycle ( $p=$ 1.9E-3) (e.g., RB1 T373, RBL2 S662, etc.) and signal transduction ( $p=1.3 \mathrm{E}-3)$ (e.g., EGFR T693, ROCK1 S1108, etc.) (Supplementary Fig. S2d - S2e). The mutation of $R B 1$ was prevalent in ESCC [26], and the mutation of EGFR was gefitinib-sensitizing mutation in ESCC [27]. In our datasets, we found the significant association between RB1 T373 and EGFR T693 (Pearson correlation, $\mathrm{R}=0.97, p$ $=1.7 \mathrm{E}-3)$ at the phosphoprotein level, indicating the co-functions in RB1 and EGFR in esophageal carcinogenesis (Supplementary Fig. S2f). The EDAC-phosphoproteins were involved in regulation of apoptosis $(p=9.9 \mathrm{E}-4)$ (e.g., APC S2093, ATM T1885, etc.). The mutation of MTOR was the driven mutation for small-bowel carcinoma, and was regarded as the potential targets in the clinic [28, 29]. In this study, we found the higher expression of MTOR ( $\mathrm{t}$-test, FC $(\mathrm{EDAC} / \mathrm{EESCC})=4.1, p=0.19)$ and MTOR S1261 (t-test, FC (EDAC/EESCC) $=4.1, p=0.04)$ in EDAC, which showed significantly positive association (Pearson correlation, $\mathrm{R}=0.96, p=2.4 \mathrm{E}-3$ ) (Fig. $\mathbf{2 g}-\mathbf{2 h}$ ). Collectively, this study presented comprehensive proteomic characterization of EESCC and EDAC at multi-omics level, and revealed the functional pathways of cell cycle in EESCC, the positive impacts of apoptosis, and metabolic processes 
in EDAC, and further demonstrated the potential co-functions of RB1 and EGFR in ESCC, and the key role of MTOR at the protein and phosphoprotein levels in EDAC.

\section{Immune-based features of EESCC and EDAC}

Recent studies have well-established the connection between inflammatory and tumorigenesis, and have considered the inflammatory is an important risk factor for gastrointestinal cancer [30]. To gain insight into features of immune infiltration of early-stage gastrointestinal cancer, we analyzed the proteomic profiles of EESCC and EDAC, and deconvoluted immune, stromal, and microenvironment cell signature using xCell (https://xcell.ucsf.edu) [31]. As a results, we found the cellular characteristics of dendritic cells (DCs) was dominant in EESCC, evidenced by the highly expressed biomarkers at the protein level, such as cluster of differentiation 14 (CD14), CD276, and CD36 (Fig. 3a - 3b; Supplementary Table S3). Furthermore, the cell signatures of endothelial cells, epithelial cells, and keratinocytes were prominent in EESCC, implying the specific characteristics of the esophagus tissues. Specifically, higher immune score (t-test, $\mathrm{FC}(\mathrm{EDAC} / \mathrm{EESCC})=1.39, p=0.04)$ and microenvironment score (t-test, FC $(\mathrm{EDAC} / \mathrm{EESCC})=1.35, p=0.03)$ were observed in EDAC, evidenced by the overrepresentation of cell signatures, such as $\mathrm{CD} 8^{+}$Tem, B cells, monocytes, neurons, and platelets (Fig. 3a). In addition, the cell markers of B cells (e.g., CD200 and CD38) and T cells (e.g., CD226 and CD81) were also overrepresented in EDAC (Fig. 3b). In EDAC, the molecules of major histocompatibility complex class I and II (MHC-I/II), were highly expressed, including HLA-B, HLA-C, HLA-E, HLA-DQA1, HLADQB1, HLA_DRA, HLA-DRB1, etc. (Fig. 3c).

The increased pro-inflammatory cytokines permit immune activity in disease [32]. To gain insight of the difference of early-stage gastrointestinal cancer, we performed cytokines-classification analysis of all identified proteins $(n=7,870)$ in early-stage gastrointestinal cancer. As a result, we identified 153 cytokines, which were classified into 8 major cytokines types as following: chemokine $(2.6 \%, n=4)$, interferon $(13.7 \%, \mathrm{n}=21)$, interleukin $(8.5 \%, \mathrm{n}=13)$, growth factor $(23.5 \%, \mathrm{n}=36)$, integrin $(20.9 \%, \mathrm{n}$ $=32)$, matrix metalloproteinase (MMP) $(3.3 \%, \mathrm{n}=5)$, tumor necrosis factor $(5.9 \%, \mathrm{n}=9)$, and $\mathrm{CD}$ $(21.6 \%, n=33)($ Fig. 3d). Comparative analysis elucidated proteins of chemokines, tumor necrosis factor, and $\mathrm{CD}$ were prevalent in EDAC, while the proteins of interferon, interleukin, growth factor, integrin, and MMP were prominent in EESCC (Fig. 3e and Supplementary Fig. S3). Specifically, the expression of IFI16 (S106 and S568), IFIT3 (S478), IRF2BP1 (S453), IRF2BPL (S69 and S547), IL1RAP (S556 and S557), ILF3 (S482 and T592), EGFR (T693 and S991), IGF2R (S2409), ICAM3 (S532), ITGA5 (S123 and S126), TRAF4 (S426), etc. was overrepresented in EESCC at the protein and phosphoprotein levels (Fig. 3f - 3g). In EDAC, the overrepresentation of the proteome expression and phosphorylation of CD44 (T720), CD300A (S260), CD2AP (T87 and S458), CD163L1 (S1402) was detected. Taken together, we elucidated the higher immune infiltration in EDAC, disclosed the cytokines classification, and revealed the specific immune features of EESCC and EDAC.

\section{Proteomic kinases profiles of EESCC and EDAC}

Human protein kinases mediate the majority of signal transduction pathways in biological processes, including cell metabolism, cell cycle, apoptosis, etc. [33], and the novel targets and inhibitors are applied to clinic strategy $[34,35]$. To access the specific kinases of early-stage gastrointestinal cancer, the identified protein kinases ( $\mathrm{n}=166$ ) were mapped to the human protein kinases. As a result, we depicted a Kinome Tree (Supplementary Fig. S4a), and found the more kinases of casein kinase 1 (CK1) group (57.1\%), cyclin-dependent/mitogen-activated protein kinase (CMGC) group (58.3\%), tyrosine-protein kinase (/receptor) (TK) group (55.3\%), and Serine/Threonine-protein kinase (/receptor) (TKL) (54.5\%) 
were detected in EESCC (Fig. 4a and Supplementary Fig. S4b). In addition, the kinases of cAMP/cGMP/calcium/phospholipid-dependent kinase (AGC) (73.9\%), serine/tyrosine/threonine protein kinase (STE) group (73.1\%), and Ca2+/CaM-dependent protein kinase (CAMK) group (67.9\%) were overrepresented in EDAC (Fig. 4b).

Specifically, CSNK1G3, ICK, CDK1, CDK2, CDK4, EGFR, FGFR1, PDGFRB, EPHB3, RAF1, BRAF, MAP3K7, ZAK, etc. were prominent in EESCC at the protein level. Consistently, the phosphorylation of CSNK1G3 T374, CDK1 T14, ICK S584, EGFR T693 and S991, LCK Y394, PDGFRB S712, EPHB3 T613, RAF1 T31 and S301, MAP3K7 S389 and ZAK S637 were overrepresented in EESCC at the phosphoprotein level (Fig. 4c - 4d). In EDAC, higher expressions of the proteome and phosphoproteome of PDPK1 (S241), PRKCD (T507), RPS6KA1 (S221), PRKACA (S339), MARK1 (S219), DAPK2 (S229), CAMK2D (T306), MAP3K2 (S239 and S153), PAK1 (S165), TNIK (T581), etc. were identified in EDAC.

To explore the functions of those kinase, we performed kinases-substates relationship analysis on the basis of kinases-substrates database [14, 16], and integrated the substrates in EESCC $(n=249)$ and EDAC $(\mathrm{n}=297)$ (Supplementary Table S4). GO-enrichment analysis of those substates revealed the elevation of cell cycle $(p=2.9 \mathrm{E}-10)$, p53 signaling pathways ( $p=5.2 \mathrm{E}-5)$, base excision repair ( $p=$ 4.6E-4), nucleotide excision repair ( $p=2.4 \mathrm{E}-3$ ), and Ras signaling pathways ( $p=1.9 \mathrm{E}-3)$ in EESCC, and disclosed the overrepresentation of mTOR signaling $(p=3.3 \mathrm{E}-5)$, PI3K-AKT signaling pathways ( $p$ $=1.4 \mathrm{E}-5)$, Mapk signaling pathways ( $p=2.6 \mathrm{E}-6)$, ErbB signaling pathways $(p=2.1 \mathrm{E}-13)$ in EDAC $($ Fig. 4e).

Kinases were applicated to the clinic strategy, such as anti-EGFR (abemaciclib) in breast cancer [36], and anti-MAP2K1 (trametinib) in melanoma [37]. We then accessed the drug targets approved by the US Food and Drug Administration database (FDA) (https://www.fda.gov) [38]. To an end, we found 6 kinases in our study were recorded in FDA datasets, in which EGFR, WEE1, CDK4, and PDGFRB were prominent in EESCC, and MAP2K1 and MAPK3 were prevalent in EDAC (Fig. 4f). Phosphorylation impacts multiple cellular processes, with site occupancy tightly regulated by the activity of kinases and phosphatases [39]. We then performed integrative analysis of the differential kinasessubstates (site), and proposed the functions of drugs approved by FDA in EESCC and EDAC. In EESCC, anti-EGFR with abemaciclib decreased the expression of EGFR (T693) and the downstream phosphorylation of GAB1 (S547), and anti-PDGFRB with imatinib in-activated the phosphorylation of PDGFRB (S712) and ABL (S210), which participated in cell cycle. Additionally, the inhibitor of ribociclib to WEE1 down-regulated the CDK1 at the protein and phosphoprotein levels, and the antiCDK4 with afatinib decreased the phosphorylation of RBL2 (S662) and RB1 (T373), resulting in the stability of cell cycle-checkpoint which was the final safeguard of genomic fidelity (Fig. 4g). In EDAC, the inhibitor of trametinib to MAP2K1 was negative associated with the phosphorylation of MAPK1 (T185) and MAPK3 (T202). Anti-MAPK3 with ulixertinib decreased the phosphorylation of AKT1 (S129), EIF4EBP1 (S35), and RPS6KA1 (S221), which down-regulated PI3K-AKT signaling and inhibited cell proliferation in EDAC (Fig. 4g). Collectively, we revealed EESCC-specific and EDACspecific kinases, elucidated the functional kinases-substates relationship network, and proposed the potential clinic strategy in EESCC and EDAC, providing a novel insight for gastrointestinal cancer in the clinic.

\section{Discussion}

Early screening and diagnosis provided better outcomes for patients, and are employed to many cancers, 
especially in gastrointestinal cancer. Great progress in mass spectrometry-based proteomics and advancement of FFPE samples enables to explore the molecular characterization of cancers, including CRC [10] gastric cancer [11] breast cancer [40] and so on. Whereas, the trace early-stage cancer sample is still a challenge, and the proteomic profiling of trace FFPE samples of early-stage cancer remain largely unknow.

In this study, we a performed comprehensive proteomic landscape of early-stage gastrointestinal cancer (EESCC and EDAC), and identified $\sim 6,000$ proteins and $>10,000$ phosphosites in single trace FFPE samples, and presented highly coverage at the protein and phosphoprotein levels, providing proteomic datasets and phosphoproteomic datasets of early-stage cancer. The consistence and positive correlation between proteome and phosphoproteome allowed us to further investigate the characterizations of early-stage gastrointestinal cancer.

The distinct separation of EESCC and EDAC indicated the tumor heterogeneity and difference among cancer types in gastrointestinal cancer. We then performed SAM [25] analysis of EESCC and EDAC, and found that primary functions of normal esophagus tissues were prominent in EESCC, such as keratinization (e.g., KRT2, KRT5, etc.), epidermis development (e.g., $\mathrm{CDH} 3, \mathrm{TCHH}$, etc.) at the protein and phosphoprotein levels. EGFR and RB1, played key roles in cell cycle, functioned in the metastasis and carcinogenesis of head and neck cancer and lung cancer [41, 42]. In EESCC, we found the high phosphorylation of EGFR T693 and RB1 T373, which showed significantly positive correlation, indicating the co-functions of EGFR and RB1 in the ESCC carcinogenesis at the phosphoprotein level. In EDAC, the high expression of metabolic proteins (e.g., ACO2, ATP5B, PFKL, etc.) and Mapk signaling pathways (e.g., MAPK1, MAPK3, ARAF, etc.) was detected, which was evidenced by the overrepresented phosphorylation of their corresponding phosphoproteins. Previous studies have proved the prominent mutations of MTOR and functions of MTOR signaling in small bowel cancer.[29] In this study, the overrepresentation of MTOR (S1261) at protein and phosphoprotein levels in EDAC, demonstrated the functions of MTOR in the carcinogenesis in duodenum cancer.

Inflammasome signaling is an emerging pillar of innate immunity, and the inflammatory microenvironment promotes gastrointestinal cancer development and invasion [43, 44]. To investigate the immune infiltration of early-stage gastrointestinal cancer, we accessed to xCell and deconvoluted the cell signature of EESCC and EDAC, and found high immune infiltration in EDAC. The cell signatures of $\mathrm{CD}^{+} \mathrm{Tem}$, B cells, monocytes, etc., were prominent in EDAC, evidenced by the overrepresentation of CD200, CD226, CD81, MHC-I (e.g., HLA-B, HLA-E, etc.) and MHC-II (e.g., HLA-DQA1, HLADRA, etc.) proteins. Cytokines played key roles in inflammatory disease. We then performed cytokines classification of all identified proteins in early-stage gastrointestinal cancer, and found the prominent of interferon, interleukin, growth factor, integrin, and MMP in EESCC, and the prevalent of chemokine, tumor necrosis factor, and CDs in EDAC both at the protein and phosphoprotein levels.

Human protein kinases participated in the majority biological processes, including cell metabolism, cell cycle, apoptosis, immune system, etc. [33], and ubiquitous in tumors, such as lung cancer, breast cancer $[45,46]$. Nowadays, kinases have become an important therapeutic target for the treatment, and inhibitors that target the kinases have been developed and are clinically active [47]. In this study, we depicted Kinome Tree of EESCC and EDAC, and found the kinases of CK1 groups, CMGC groups, TK groups, and TKL groups were overrepresented in EESCC, and the kinases of AGC group, CAMK group, and STE group were prevalent in EDAC at the protein and phosphoprotein levels. In addition, the kinases-substrates correlation network revealed the positive impacts of cell cycle, p53 signaling pathways, and DNA repair in EESCC, and the positive impacts of PI3K-AKT signaling pathways, mTOR 
signaling pathways, Mapk signaling pathways in EDAC. We also proposed the potential functional mechanism of drugs approved by FDA in EESCC and EDAC. For example, the inhibitor of trametinib to MAP2K1 decreased the phosphorylation of MAPK1 (T185) and MAPK3 (T202), and anti-MAPK3 with ulixertinib had negative impacts on the phosphorylation of AKT1 (S129), EIF4EBP1 (S35), and RPS6KA1 (S221), which down-regulated PI3K-AKT signaling and inhibited cell proliferation in EDAC. However, limits still existed in our study. The more samples were need, and the trace amount samples restricted to collected genomic and transcriptomic data, and so on.

\section{Conclusion}

This study presented a comprehensive proteomic landscape for early-stage gastrointestinal cancer for the first time, with identification of $\sim 6,000$ proteins and $>10,000$ phosphosites in single trace FFPE sample. We revealed the functional classification of all identified proteins and phophproteins in EESCC and EDAC. We also disclosed the distinct separation between EESCC and EDAC, and illustrating the impacts of cell cycle in EESCC, and of apoptosis and metabolic processes in EDAC at the protein and phosphoprotein levels. In addition, we deconvoluted the immune infiltration of early-stage gastrointestinal cancer, and found higher immune cell signatures in EDAC. Additionally, we revealed the specific cytokines in EESCC and EDAC. Furthermore, we delineated the Kinome Tree of EESCC and EDAC, elucidated the specific kinases, and proposed the potential clinic strategy in EESCC and EDAC, delivering a novel insight of the clinic therapeutic strategy for gastrointestinal cancer.

\section{Authors' contributions}

Lingling Li and Chen Ding conceived and designed the analysis: Lingling Li and Hui Liu collected the data. Lingling Li, Hui Liu, Yan Li, Chunmei Guo, Bing Wang, Dan Shen and Qiao Zhang contributed analysis tools. Lingling Li performed the analysis. Lingling Li and Chen Ding wrote the paper.

\section{Funding}

This study was supported by Chinese Ministry of Science and Technology (2016YFA0502500), and Key Projects of Strategic International Scientific and Technology Innovation Cooperation of Chinese Ministry of Science and Technology (2020YFE0201600).

\section{Availability data and materials}

All the proteomic/phosphoproteomic raw data and the results files of EESCC and EDAC had been uploaded to the iProx Consortium (https://www.iprox.org/) with the PXD identifiers (PXD026736).

\section{Declarations}

\section{Ethics approval and consent to participate}

The study was carried out in compliance with the ethical standards of Helsinki Declaration II and approved by the Institution Review Board of Fudan University Zhongshan Hospital (B2019-200R)

\section{Consent for publication}

No applicable.

\section{Competing interests}

The authors declare that they have no competing interest.

\section{Author details}

${ }^{1}$ State Key Laboratory of Genetic Engineering and Collaborative Innovation Center for Genetics and 
Development, School of Life Sciences, Institute of Biomedical Sciences, Human Phenome Institute, Fudan University, Shanghai 200433, China;

${ }^{2}$ State Key Laboratory Cell Differentiation and Regulation, Overseas Expertise Introduction Center for Discipline Innovation of Pulmonary Fibrosis, (111 Project), College of Life Science, Henan Normal University, Xinxiang, Henan 453007, China.

\section{References}

1. Bray, F., et al., Global cancer statistics 2018: GLOBOCAN estimates of incidence and mortality worldwide for 36 cancers in 185 countries. CA Cancer J Clin, 2018. 68(6): p. 394-424.

2. $\quad$ Siegel, R.L., et al., Cancer Statistics, 2021. CA Cancer J Clin, 2021. 71(1): p. 7-33.

3. Enzinger, P.C. and R.J. Mayer, Medical progress - Esophageal cancer. New England Journal of Medicine, 2003. 349(23): p. 2241-2252.

4. Yamamoto, H., et al., Successful en bloc resection of a large superficial gastric cancer by using sodium hyaluronate and electrocautery incision forceps. Gastrointestinal Endoscopy, 2001. 54(5): p. 629-632.

5. Song, W.C., X.L. Qiao, and X.Z. Gao, A comparison of endoscopic submucosal dissection (ESD) and radical surgery for early gastric cancer: a retrospective study. World J Surg Oncol, 2015. 13: p. 309.

6. Jiang, D., et al., A retrospective study of endoscopic resection for 368 patients with early esophageal squamous cell carcinoma or precancerous lesions. Surg Endosc, 2017. 31(5): p. 2122-2130.

7. Wani, S., et al., Comparison of endoscopic therapies and surgical resection in patients with early esophageal cancer: a population-based study. Gastrointest Endosc, 2014. 79(2): p. 224$232 \mathrm{e} 1$.

8. Zhong, J., et al., Enhanced and controlled chromatin extraction from FFPE tissues and the application to ChIP-seq. BMC Genomics, 2019. 20(1): p. 249.

9. Zhu, Y., et al., High-throughput proteomic analysis of FFPE tissue samples facilitates tumor stratification. Mol Oncol, 2019. 13(11): p. 2305-2328.

10. Vasaikar, S., et al., Proteogenomic Analysis of Human Colon Cancer Reveals New Therapeutic Opportunities. Cell, 2019. 177(4): p. 1035-1049 e19.

11. Ge, S., et al., A proteomic landscape of diffuse-type gastric cancer. Nat Commun, 2018. 9(1): p. 1012.

12. Kanehisa, M. and S. Goto, KEGG: kyoto encyclopedia of genes and genomes. Nucleic Acids Res, 2000. 28(1): p. 27-30.

13. Casado, P., et al., Phosphoproteomics data classify hematological cancer cell lines according to tumor type and sensitivity to kinase inhibitors. Genome Biology, 2013. 14(4).

14. Hornbeck, P.V., et al., Phosphosite: A bioinformatics resource dedicated to physiological protein phosphorylation. Proteomics, 2004. 4(6): p. 1551-1561.

15. Dinkel, H., et al., Phospho.ELM: a database of phosphorylation sites-update 2011. Nucleic Acids Research, 2011. 39: p. D261-D267.

16. Yang, C.Y., et al., PhosphoPOINT: a comprehensive human kinase interactome and phosphoprotein database. Bioinformatics, 2008. 24(16): p. I14-I20.

17. Schwartz, D. and S.P. Gygi, An iterative statistical approach to the identification of protein phosphorylation motifs from large-scale data sets. Nature Biotechnology, 2005. 23(11): p. 
1391-1398.

18. Casado, P., et al., Kinase-Substrate Enrichment Analysis Provides Insights into the Heterogeneity of Signaling Pathway Activation in Leukemia Cells. Science Signaling, 2013. 6(268).

19. Hornbeck, P.V., et al., PhosphoSitePlus, 2014: mutations, PTMs and recalibrations. Nucleic Acids Research, 2015. 43(D1): p. D512-D520.

20. Jiang, Y., et al., Proteomics identifies new therapeutic targets of early-stage hepatocellular carcinoma. Nature, 2019. 567(7747): p. 257-+.

21. Schwanhausser, B., et al., Global quantification of mammalian gene expression control (vol 473, pg 337, 2011). Nature, 2013. 495(7439): p. 126-127.

22. Schwanhausser, B., et al., Global quantification of mammalian gene expression control. Nature, 2011. 473(7347): p. 337-342.

23. Yazdian-Robati, R., et al., Comparative proteome analysis of human esophageal cancer and adjacent normal tissues. Iranian Journal of Basic Medical Sciences, 2017. 20(3): p. 265-271.

24. Pawar, H., et al., Quantitative tissue proteomics of esophageal squamous cell carcinoma for novel biomarker discovery. Cancer Biology \& Therapy, 2011. 12(6): p. 510-522.

25. Tusher, V.G., R. Tibshirani, and G. Chu, Significance analysis of microarrays applied to the ionizing radiation response. Proc Natl Acad Sci U S A, 2001. 98(9): p. 5116-21.

26. Song, Y.M., et al., Identification of genomic alterations in oesophageal squamous cell cancer. Nature, 2014. 509(7498): p. 91-+.

27. Guo, M.Z., et al., Gefitinib-sensitizing mutation in esophageal carcinoma cell line Kyse450. Cancer Biology \& Therapy, 2006. 5(2): p. 152-155.

28. Schrock, A.B., et al., Genomic Profiling of Small-Bowel Adenocarcinoma. Jama Oncology, 2017. 3(11): p. 1546-1553.

29. Banck, M.S., et al., The genomic landscape of small intestine neuroendocrine tumors. Journal of Clinical Investigation, 2013. 123(6): p. 2502-2508.

30. Terzic, J., et al., Inflammation and Colon Cancer. Gastroenterology, 2010. 138(6): p. 2101-U119.

31. Aran, D., Z. Hu, and A.J. Butte, xCell: digitally portraying the tissue cellular heterogeneity landscape. Genome Biol, 2017. 18(1): p. 220.

32. Huang, C.L., et al., Clinical features of patients infected with 2019 novel coronavirus in Wuhan, China. Lancet, 2020. 395(10223): p. 497-506.

33. Fedorov, O., S. Muller, and S. Knapp, The (un)targeted cancer kinome. Nat Chem Biol, 2010. 6(3): p. 166-169.

34. Bradshaw, J.M., et al., Prolonged and tunable residence time using reversible covalent kinase inhibitors. Nature Chemical Biology, 2015. 11(7): p. 525-+.

35. Ferguson, F.M. and N.S. Gray, Kinase inhibitors: the road ahead. Nat Rev Drug Discov, 2018. 17(5): p. 353-377.

36. Goetz, M.P., et al., MONARCH 3: Abemaciclib As Initial Therapy for Advanced Breast Cancer. Journal of Clinical Oncology, 2017. 35(32): p. 3638-+.

37. Flaherty, K.T., et al., Improved Survival with MEK Inhibition in BRAF-Mutated Melanoma. New England Journal of Medicine, 2012. 367(2): p. 107-114.

38. Momper, J.D., et al., Adolescent Dosing and Labeling Since the Food and Drug Administration Amendments Act of 2007. Jama Pediatrics, 2013. 167(10): p. 926-932.

39. Ubersax, J.A. and J.E. Ferrell, Mechanisms of specificity in protein phosphorylation. Nature 
Reviews Molecular Cell Biology, 2007. 8(7): p. 530-541.

40. Xu, J.Y., et al., Integrative Proteomic Characterization of Human Lung Adenocarcinoma. Cell, 2020. 182(1): p. 245-261 e17.

41. Beck, T.N., et al., EGFR and RBI as Dual Biomarkers in HPV-Negative Head and Neck Cancer. Molecular Cancer Therapeutics, 2016. 15(10): p. 2486-2497.

42. Marcoux, N., et al., EGFR-Mutant Adenocarcinomas That Transform to Small-Cell Lung Cancer and Other Neuroendocrine Carcinomas: Clinical Outcomes. Journal of Clinical Oncology, 2019. 37(4): p. 278-+.

43. Man, S.M., Inflammasomes in the gastrointestinal tract: infection, cancer and gut microbiota homeostasis. Nature Reviews Gastroenterology \& Hepatology, 2018. 15(12): p. 721-737.

44. Echizen, K., et al., The inflammatory microenvironment that promotes gastrointestinal cancer development and invasion. Adv Biol Regul, 2018. 68: p. 39-45.

45. Smits, A.J.J., et al., The Frequency of Egfr and Kras Mutations in Non-Small Cell Lung Carcinomas in the Dutch Population. Journal of Thoracic Oncology, 2011. 6(6): p. S1528S1528.

46. Villarino, A.V., Y. Kanno, and J.J. O'Shea, Mechanisms and consequences of Jak-STAT signaling in the immune system. Nature Immunology, 2017. 18(4): p. 374-384.

47. Roengvoraphoj, M., et al., Epidermal growth factor receptor tyrosine kinase inhibitors as initial therapy for non-small cell lung cancer: Focus on epidermal growth factor receptor mutation testing and mutation-positive patients. Cancer Treatment Reviews, 2013. 39(8): p. 839-850.

\section{Figure Legends:}

Fig. 1. Proteomic landscape of trace FFPE samples in EESCC and EDAC. a Overview of the experimental design. A total of 12 samples were collected from EESCC ( 3 cases) and EDAC ( 3 cases). b Proteomic landscape of EESCC. $\mathbf{c}$ Proteomic landscape of EDAC. The highest and lowest abundance proteins were showed in the box. $\mathbf{d}$ The number of identified proteins in EESCC (purple) and EDAC (red). e Phosphoproteomic profiles of EESCC. f Phosphoproteomic profiles of EDAC. The highest and lowest abundance proteins were showed in the box. $\mathbf{g}$ The number of identified proteins in EESCC (purple) and EDAC (red).

Fig. 2. Proteomic characterization of EESCC and EDAC. a Spearman's correlation coefficients among 6 gastrointestinal cancer samples. b PCA analysis showing distinct separation between EESCC and EDAC at the protein (left) and phosphoprotein levels. $\mathbf{c}$ Volcano analysis depicted the differential expressed proteins of EESCC and EDAC. $\mathbf{d}$ Bar chart presenting the functional pathways in EESCC (top) and EDAC (bottom). e Proteins in functional pathways that were differentially expressed in EESCC and EDAC at protein and phosphoprotein levels. $\mathrm{A} A$ brief of the differential proteins and functional pathways in EESCC (top) and EDAC (bottom). g Boxplot showing MTOR was highly expressed in EDAC at the protein (left) and phosphoprotein (right) levels. h Pearson's correlation coefficients indicated significantly positive association between MTOR proteome and phosphoproteome (S1261).

Fig. 3. Immune-based features of EESCC and EDAC. a Heatmap showing the immune infiltration of EESCC and EDAC at the protein level. Top: heatmap showing the immune and microenvironment score. Bottom: heatmap showing the cell signatures. $\mathbf{b}$ The proteome-level expression of the biomarkers of DCs, $\mathrm{B}$ cells, and T cells in EESCC and EDAC. $\mathbf{c}$ Expression of MHC-I (top) and MHC-II (bottom) in EESCC 
and EDAC at the protein level. $\mathbf{d}$ Pie chart showing eight major classifications of cytokines identified in early-stage gastrointestinal cancer. e Bar charts illustrating the proportion of eight major classifications of cytokines in ESSCC (purple) and EDAC (red). f Heatmap showing the expression of the proteins of the major cytokines in EESCC and EDAC at the protein level. $g$ Heatmap showing the expression of the proteins of the major cytokines in EESCC and EDAC at the phosphoprotein level.

Fig. 4. Proteomic kinases profiles in EESCC and EDAC. a Distribution and the number of the seven major kinases types in EESCC (purple) and EDAC (red). b Bar charts showing the differential proportion of major kinases types in EESCC (purple) and EDAC (red). $\mathbf{c}$ Heatmap representing the expression of the proteins of major kinases types in EESCC and EDAC at the protein level. d Heatmap representing the expression of the proteins of major kinases types in EESCC and EDAC at the phosphoprotein level. e Enrichment of the kinases and the downstream substates showing the dominant pathways in EESCC (purple) and EDAC (red). $P$ value $<0.05$ and FDR q value $<0.3$ were considered as significant enrichment. f Heatmap showing the expression of the inhibitors (FDA drug) to kinases (top) (proteome) and the kinases regulated substates (phosphoproteome) in EESCC and EDAC. g Pathways based on the selected phospho-substates and kinases, with relevant drugs shown by targets in EESCC (left) and EDAC (right).

Supplementary Fig. S1. Overview of the proteomic profiles of EESCC and EDAC. a Schematic illustration of the samples and experimental workflow. $\mathbf{b}$ The number of the patient cases, identified proteins, and phosphosites in EESCC and EDAC. $\mathbf{c}$ Venn diagram showing the overlaps of the identified proteins (left) and phosphosites (right) in 3 EESCC patients. d Venn diagram showing the overlaps of the identified proteins (left) and phosphosites (right) in 3 EDAC patients.

Supplementary Fig. S2. Phosphoproteomic characteristics of EESCC and EDAC phosphoproteome. a Venn diagram representing the overlaps of the identification (left) and Pearson's correlation coefficients (right) between proteome and phosphoproteome in EESCC. b Venn diagram representing the overlaps of the identification (left) and Pearson's correlation coefficients (right) between proteome and phosphoproteome in EDAC. $\mathbf{c}$ Volcano analysis depicted the differential phosphorylation of EESCC and EDAC. d Bar chart showing the functional pathways in EESCC (top) and EDAC (bottom) phosphoproteome. e Phosphoproteins in functional pathways that were differentially expressed in EESCC (left) and EDAC (right) at protein and phosphoprotein levels. f Pearson's correlation coefficients indicated significantly positive association between RB1 (T3730 and EGFR (T693).

Supplementary Fig. S3. Eight major classifications of cytokines identified in early-stage gastrointestinal cancer. All the classified types (left) and the proteins of the cytokines identified in EESCC (middle) and EDAC (right) were shown.

Supplementary Fig. S4. Major kinases in EESCC and EDAC. a A Kinome Tree of EESCC (purple) and EDAC (red) mapped to human kinases datasets. $\mathbf{b}$ The distribution of the major kinases types in EESCC (left) and EDAC (right). 
a

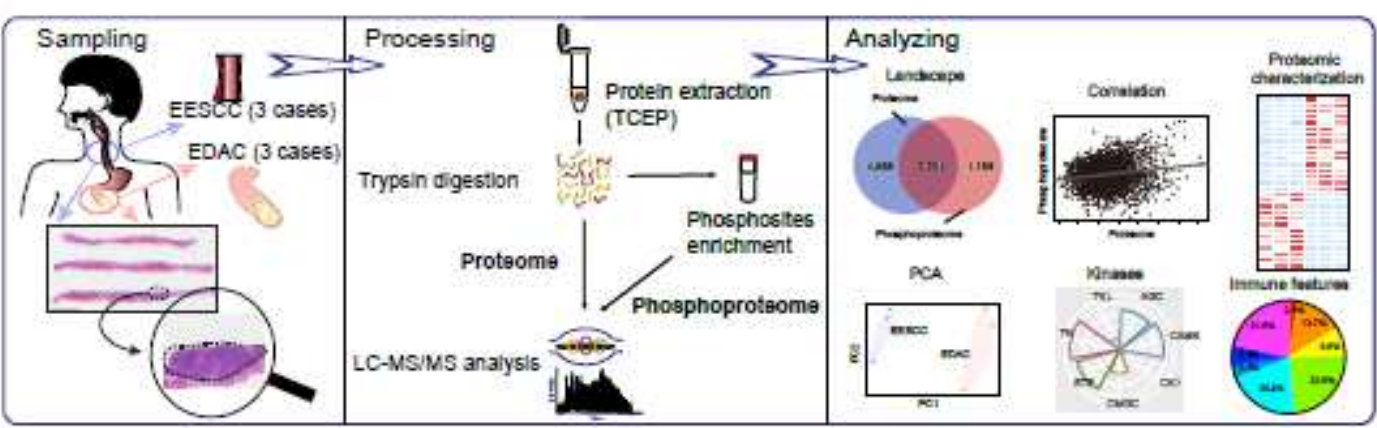

b

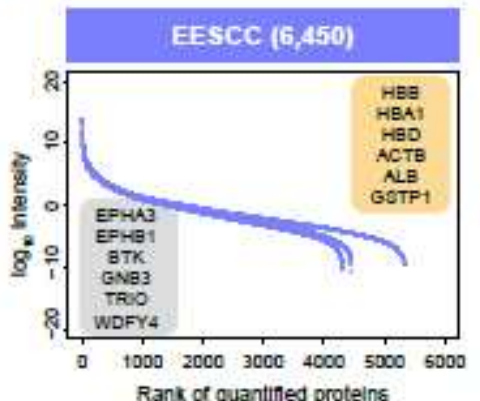

e

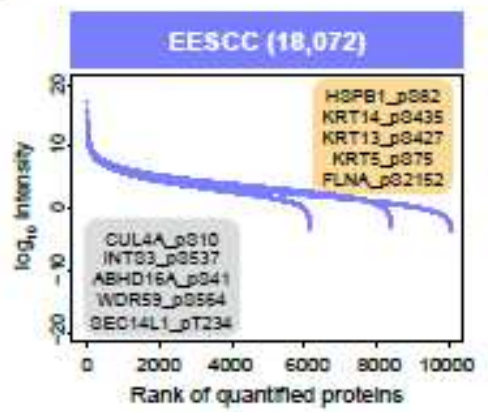

c

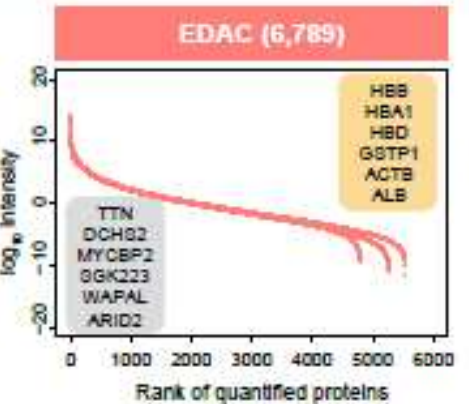

f

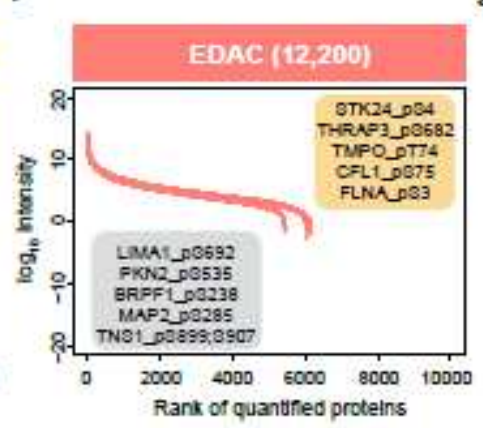

d

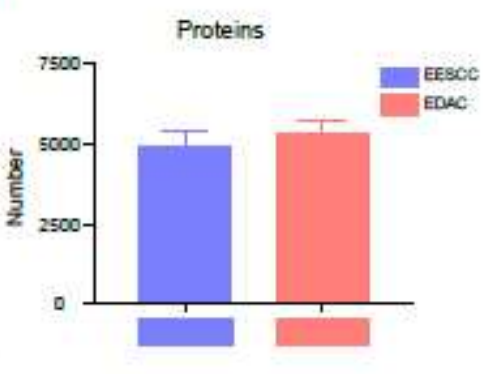

g Phosphosites

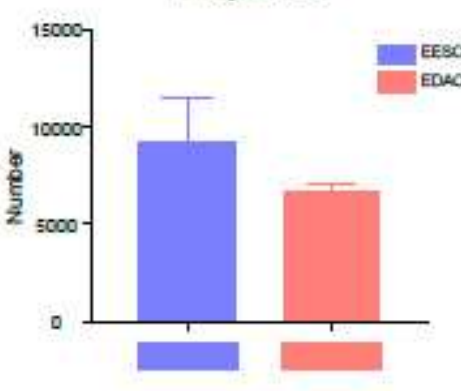

\section{Figure 1}

Proteomic landscape of trace FFPE samples in EESCC and EDAC. a Overview of the experimental design. A total of 12 samples were collected from EESCC (3 cases) and EDAC (3 cases). b Proteomic landscape of EESCC. c Proteomic landscape of EDAC. The highest and lowest abundance proteins were showed in the box. $d$ The number of identified proteins in EESCC (purple) and EDAC (red). e Phosphoproteomic profiles of EESCC. $f$ Phosphoproteomic profiles of EDAC. The highest and lowest abundance proteins were showed in the box. $g$ The number of identified proteins in EESCC (purple) and EDAC (red). 

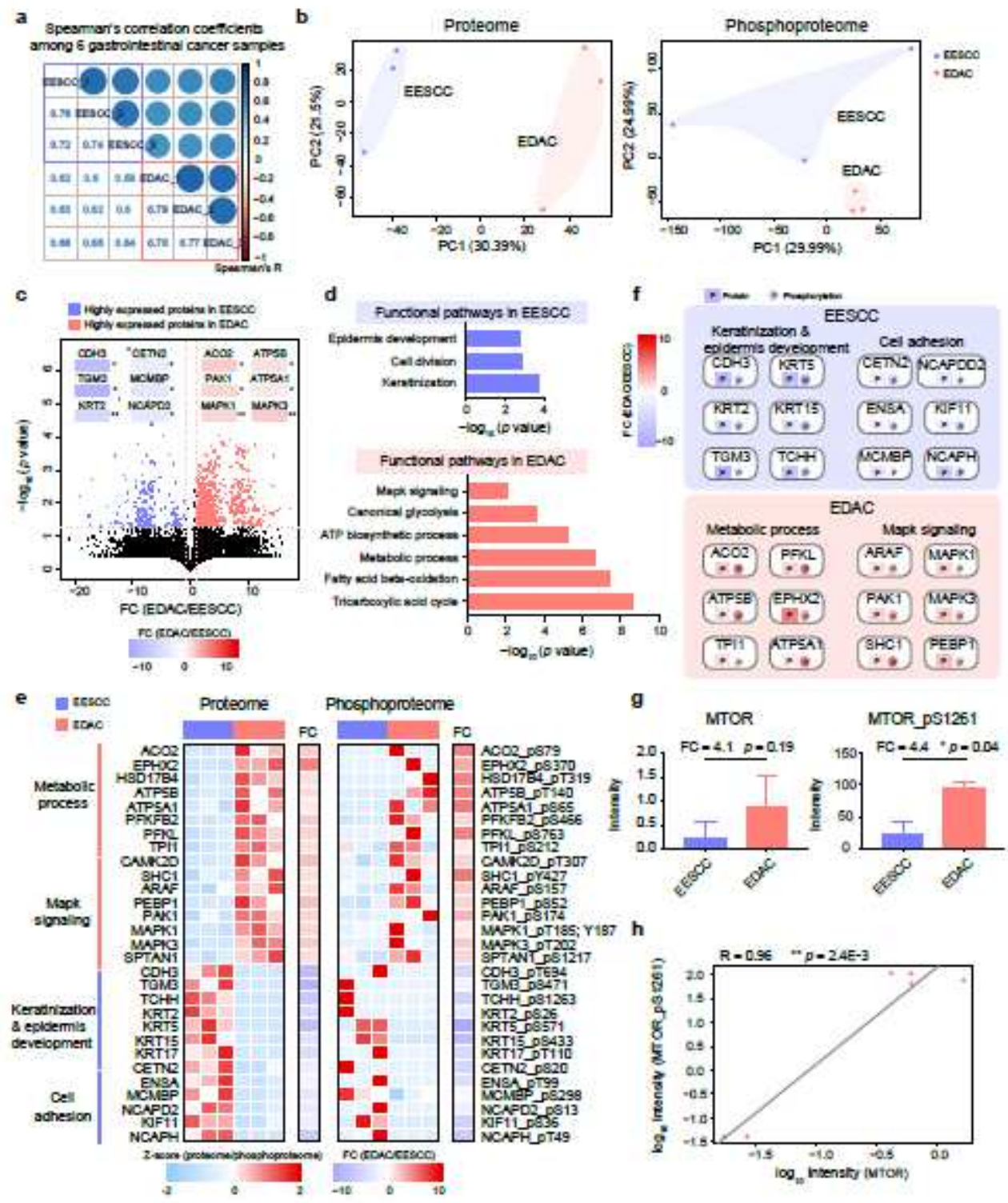

Figure 2

Proteomic characterization of EESCC and EDAC. a Spearman's correlation coefficients among 6 gastrointestinal cancer samples. b PCA analysis showing distinct separation between EESCC and EDAC at the protein (left) and phosphoprotein levels. c Volcano analysis depicted the differential expressed proteins of EESCC and EDAC. $d$ Bar chart presenting the functional pathways in EESCC (top) and EDAC (bottom). e Proteins in functional pathways that were differentially expressed in EESCC and EDAC at protein and phosphoprotein levels. $f$ A brief of the differential proteins and functional pathways in EESCC (top) and EDAC (bottom). g Boxplot showing MTOR was highly expressed in EDAC at the protein (left) and phosphoprotein (right) levels. h Pearson's correlation coefficients indicated significantly positive association between MTOR proteome and phosphoproteome (S1261). 


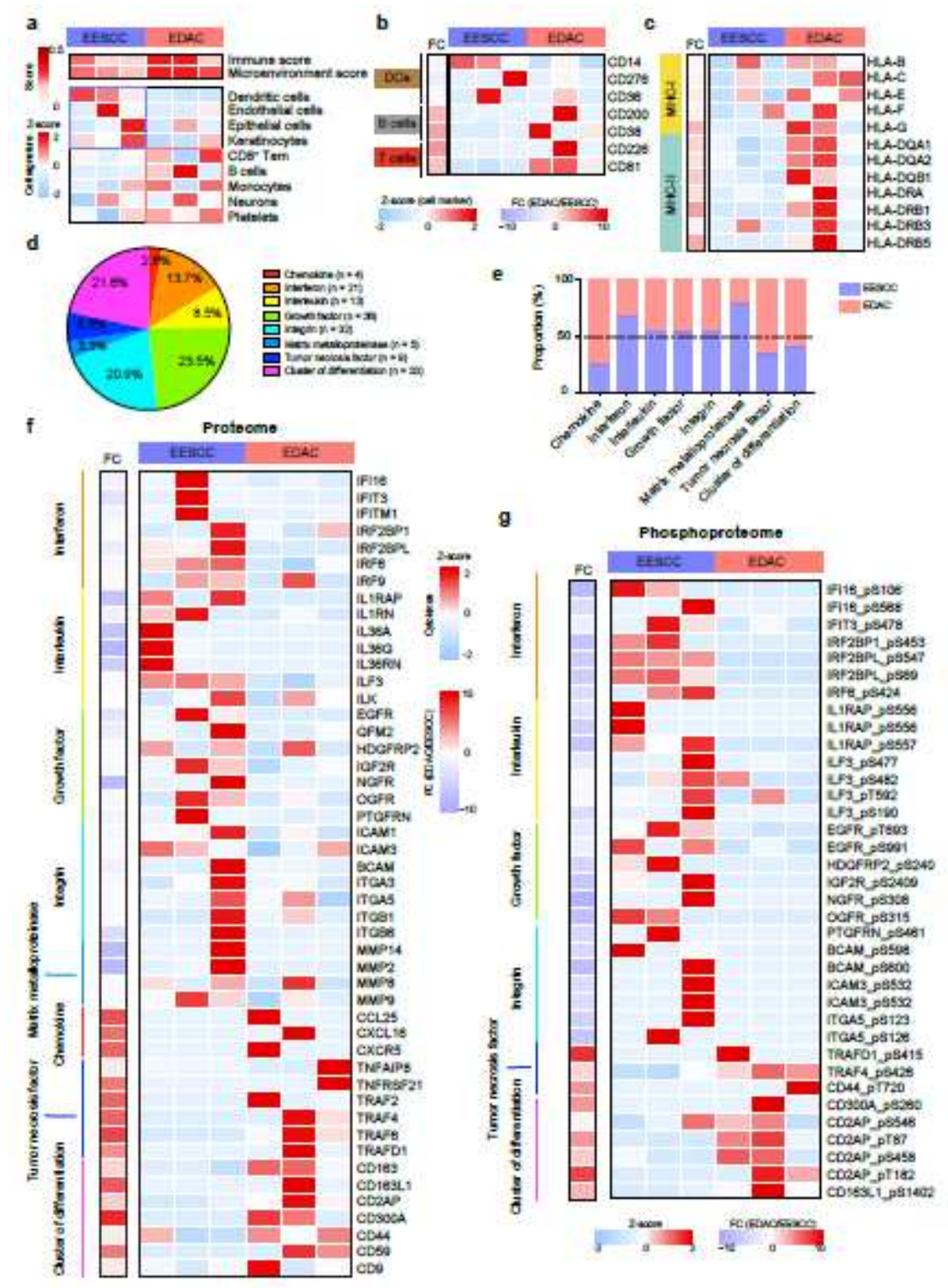

Figure 3

Immune-based features of EESCC and EDAC. a Heatmap showing the immune infiltration of EESCC and EDAC at the protein level. Top: heatmap showing the immune and microenvironment score. Bottom: heatmap showing the cell signatures. $b$ The proteome-level expression of the biomarkers of DCs, $B$ cells, and T cells in EESCC and EDAC. c Expression of MHC-I (top) and MHC-II (bottom) in EESCC and EDAC at the protein level. $d$ Pie chart showing eight major classifications of cytokines identified in early-stage gastrointestinal cancer. e Bar charts illustrating the proportion of eight major classifications of cytokines in ESSCC (purple) and EDAC (red). $f$ Heatmap showing the expression of the proteins of the major cytokines in EESCC and EDAC at the protein level. g Heatmap showing the expression of the proteins of the major cytokines in EESCC and EDAC at the phosphoprotein level. 


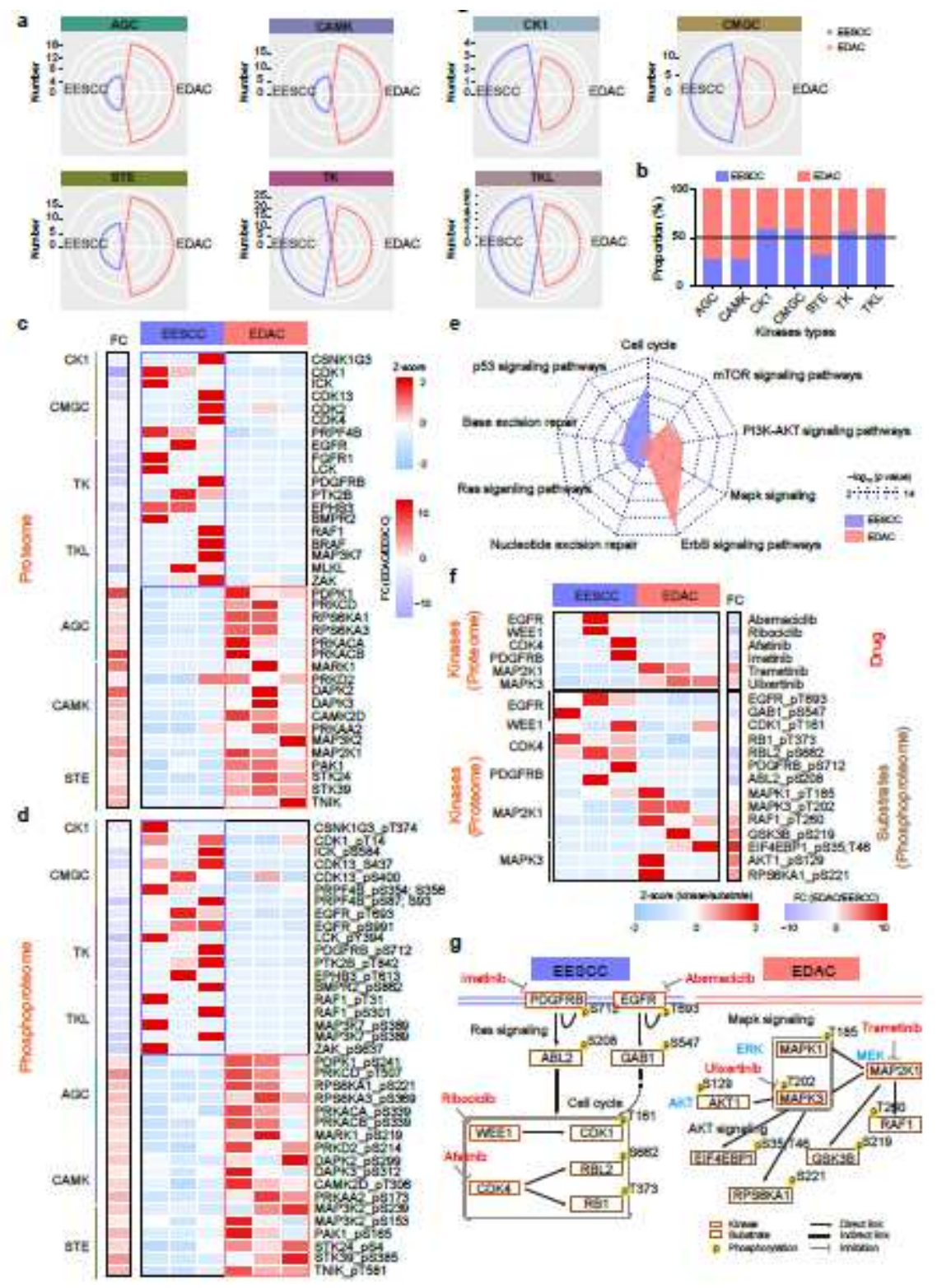

Figure 4

Proteomic kinases profiles in EESCC and EDAC. a Distribution and the number of the seven major kinases types in EESCC (purple) and EDAC (red). b Bar charts showing the differential proportion of major kinases types in EESCC (purple) and EDAC (red). c Heatmap representing the expression of the proteins of major kinases types in EESCC and EDAC at the protein level. $d$ Heatmap representing the expression of the proteins of major kinases types in EESCC and EDAC at the phosphoprotein level. e Enrichment of the kinases and the downstream substates showing the dominant pathways in EESCC (purple) and EDAC (red). $P$ value $<0.05$ and FDR q value $<0.3$ were considered as significant enrichment. f Heatmap showing the expression of the inhibitors (FDA drug) to kinases (top) (proteome) and the kinases regulated substates (phosphoproteome) in EESCC and EDAC. g Pathways based on the selected phospho-substates and kinases, with relevant drugs shown by targets in EESCC (left) and EDAC (right).

\section{Supplementary Files}


This is a list of supplementary files associated with this preprint. Click to download.

- SupplementaryFigure.pdf

- Graphicalabstract.pdf

- SupplementaryTableS1.xlsx

- SupplementaryTableS2.xlsx

- SupplementaryTableS3.xlsx

- SupplementaryTableS4.xlsx 